\title{
NEW IXORA SPECIES FROM THE SOLOMON ISLANDS
}

\author{
C. E. B. Bremekamp
}

The Melanesian species of Ixora L. belong partly to the subgenus Phylleilema Asa Gray, which finds its main development in this area, and partly to Pavetropsis Brem., a section of the subgenus Pavetroides Brem. The subgenus Phylleilema is easily recognizable by its fewflowered, subcapitate inflorescence enclosed between two large foliar bracts; PAvetToIDEs on the other hand has a corymbose or paniculate inflorescence, sometimes with a few fairly conspicuous bracts at its base, but the latter are never as large as in Phylleilema, and they are moreover stipular, not foliar. PAvetropsis is the largest and most widespread section of this subgenus: it ranges from the Seychelles to Melanesia. The subsessile, trichotomous corymb, the absence of articulations in the latter's peripheral part, and the naked corolla throat distinguish it from the other sections. The three species from the Solomon Islands which I will presently describe belong to this group.

The species of the Solomon Islands differ rather conspicuously from the species of the Malay Archipelago, with which I have dealt at a former occasion (Bull. Jard. Bot. Buitenz. Sér. III, 14: 231-234, 314-335. 1937). The latter could be grouped in three series: Glabristylae, PiloSistylae and Orientales. The species of the Solomon Islands agree in the pilose styles with those belonging to the two last series; from the Orientales, which occupy the region to the west of the Solomon Islands, they differ in the possession of short, but well developed calyx lobes; from all three, however, they are separated by the rather small size of the stipular bracts at the base and at the top of the peduncle, by the opposite position, not only of the branchlets of the first order, but of those of the second order too, and by the arrangement of the flowers in fairly distinct triads. In these characters they agree on the one hand with the section Pogonantuus Brem., from which they differ however conspicuously in the completely glabrous corolla throat, and on the other hand with the species of the Fijian Islands and probably also with those of New Caledonia. The descriptions of the latter, however, are not sufficiently detailed, and as I had no material for comparison, I am at present unable to decide their position. Two of the three species described below are characterized moreover by the presence of well developed bracteoles at the base of the ovary, and by a two-seeded, but nevertheless nearly globose drupe: in other species the drupe is as a rule 
flattened or more or less distinctly didymous when both seeds are developed.

1. Ixora ysabellae, sp. nov.; Typus: L. J. Brass 3525 in herb. Arb. Arnold. Forma et glabrescentia foliorum, floribus pedicellatis, haud dense bracteatis, calyce breviter lobato ad I. myrtifoliam A. C. Smith vergens, statura minore, foliis longioribus, apice caudato-acuminatis, inflorescentia majore, pedicellis longioribus ab ea satis distincta.

Arbuscula 1.5-2 m. alta, parce ramosa. Rami novelli primum complanati et leviter bisulcati, mox teretes tamen, glabri, opaci, 3.5-4 mm. diam., deinde cortice griseo-brunneo vestiti. Folia glabra petiolo crasso $4 \mathrm{~mm}$. longo; lamina oblanceolata plerumque circ. $25 \mathrm{~cm}$. longa et $8 \mathrm{~cm}$. lata, apice caudato-acuminata, basi subcordata, coriacea, supra nitidula, sicc. supra badia et subtus ferruginea, margine subrevoluta, costa crassa subtus valde prominente et ibidem distincte carinata, nervis utroque latere costae plerumque 13-15 subtus prominulis, venulis laxe reticulatis subtus colore saturatiore conspicuis. Stipulae basi breviter connatae, late triangulares et in aristam vaginae aequilongam exeuntes, $8 \mathrm{~mm}$. longae. Inflorescentia foliis magnitudine paulum reductis, $15 \mathrm{~cm}$. longis praecessa, subsessilis, trichotome corymbosa, $4-4.5 \mathrm{~cm}$. alta, $10-11 \mathrm{~cm}$. diam., floribus circ. 200, puberula; pedunculus $3 \mathrm{~mm}$. longus; ramuli infimi et ramuli jugi secundi basi oppositi et subarticulati; ramuli alii suboppositi; triades subdistinctae; internodium basale axis $12 \mathrm{~mm}$, internodia basalia ramulorum infimorum $28 \mathrm{~mm}$; internodia alia peripheriam versus gradatim longitudine decrescentia; pedicelli $1-3 \mathrm{~mm}$; ramuli infimi foliis rudimentariis lineari-lanceolatis $5 \mathrm{~mm}$. longis quae stipulis normalibus $6 \mathrm{~mm}$. longis connata suffulti; alii bracteis filiformibus quorum infimae interdum stipulis sejunctis munitae et linea elevata connectae, superiores in ramulos pedicellosque interdum alte egredientes; bracteolae basi ovarii insertae lineari-triangulares et ovario paulo breviores. Flores ovario calyceque puberulis. Calyx ovario aequilongus, tubo $0.3 \mathrm{~mm}$. longo, lobis ovatis acutis $1.2 \mathrm{~mm}$. longis. Corolla alba, extus intusque glabra, tubo $9 \mathrm{~mm}$. longo et $1.2 \mathrm{~mm}$. diam., lobis linearibus $7 \mathrm{~mm}$. longis et $1.5 \mathrm{~mm}$. latis, acutis. Stamina $6 \mathrm{~mm}$. longa, filamentis glabris $2.5 \mathrm{~mm}$. longis, antheris apiculatis $5 \mathrm{~mm}$. longis. Stylus ad medium pilosus, stigmatibus $3 \mathrm{~mm}$. longis comprehensis $14 \mathrm{~mm}$. longus. Drupa globosa, rubra, glabra, $7.5 \mathrm{~mm}$. diam., biseminalis, calycis lobis persistentibus haud conspicue coronata.

Solomon Islands: Y s a b e l I s l a n d, Tataba, alt. 50 m., in dense rain forest, common, leg. L. J. Brass 3525, Jan. 1933 (Arnold Arb.).

In the form of the leaves and of the inflorescence, in the pedicellate, not copiously bracteate flowers, and in the comparatively short calyx 
lobes this species resembles I. myrtifolia A. C. Smith and I. maxima Seem. From the latter it is easily distinguished by its glabrous and much smaller leaves, and from I. myrtifolia by its larger leaves and its larger and laxer inflorescence.

2. Ixora bougainvilliae, sp. nov.; Typus: S. F. Kajewski 1993 in herb. Arb. Arnold. Ad speciem precedentem et ad I. myrtifolium A. C. Smith vergens, sed foliis longius petiolatis, basi acutis an subacutis, stipulis longioribus ab utraque faciliter distinguenda.

Arbor usque ad $20 \mathrm{~m}$. alta. Rami novelli profunde bisulcati, papillosiores, opaci, 3.5-4 mm. diam.; vetustiores validiores, cortice griseo opaco vestiti. Folia glabra, petiolo crasso $10-17 \mathrm{~mm}$. longo; lamina oblanceolata, 25-40 cm. longa et $7.5-11 \mathrm{~cm}$. lata, apice caudato-acuminata, basi acuta an subacuta, coriacea, utrimque opaca, sicc. olivacea, margine subrevoluta, costa crassa subtus valde prominente et ibidem distincte carinata, nervis utroque latere costae 10-15 supra canaliculatis et subtus prominulis, reticulatione densa utrimque distinguenda. Stipulae triangulares, basi breviter connatae, in aristam validam exeuntes, $1 \mathrm{~cm}$. longae. Inflorescentia breviter pedunculata, trichotome corymbosa, 4.2 $\mathrm{cm}$. alta et $11 \mathrm{~cm}$. diam., floribus circ. 200, peripheriam versus puberula; pedunculus $6 \mathrm{~mm}$. longus; ramuli infimi basi articulati et sicut ramuli jugi secundi oppositi et patentissimi; ramuli alii haud distincte oppositi; triades plerumque difficiliter distinguendae; internodium basale axis 13 mm.; internodia basalia ramulorum infimorum $27 \mathrm{~mm}$.; internodia alia peripheriam versus gradatim longitudine decrescentia; pedicelli $1-2 \mathrm{~mm}$; ramuli infimi foliis rudimentariis deciduis stipulas normales parvas i.e. $5 \mathrm{~mm}$. longas exhibentibus suffulti; alii bracteis triangularibus parvis quarum superiores in ramulos pedicellosque egrediuntur; bracteolae basi ovarii insertae, triangulares, ovario subaequilongae. Flores ovario calyceque puberulis. Calyx ovario aequilongus, tubo $0.2 \mathrm{~mm}$., lobis ovatis $1.0 \mathrm{~mm}$. longis acutis. Corolla ignota. Drupa depresse globosa, scarlatina, vivo $1 \mathrm{~cm}$. alta et $1.4 \mathrm{~cm}$. diam., sicc. $7 \mathrm{~mm}$. alta et $9 \mathrm{~mm}$. diam., biseminalis, calycis lobis persistentibus haud conspicue coronata.

Solomon Islands: B o u g a i n vill e I s l a n d, Koniguru, Buin, alt. 800 m., in rain forest, common, leg. S. F. Kajewski 1993, Aug. 1933 (Arnold Arb.).

Vern. name: "katioloba."

By the large size of the leaves this species reminds one of $I$. maxima Seem., from which it differs, however, conspicuously in its glabrousness. From I. ysabellae Brem. and I. myrtifolia A. C. Smith it is easily distinguished by its habit (it is a large tree) and in the shape and size of the leaves. 
3. Ixora solomonensium, sp. nov.; Typus: L. J. Brass 2948 in herb. Arb. Arnold. Habitu I. timorensi Decn. similior, sed corolla fauce glaberrima faciliter ab ea distinguenda; inter species sectionis PAvETTOPsis maxime ad I. elegantem Gillespie et ad I. tubifloram A. C. Smith vergens, ab I. eleganti calyce glabro, brevius lobato, ab I. tubiflora absentia bracteolarum et corolla multo breviore distinguenda.

Arbor usque ad $15 \mathrm{~m}$. alta. Rami novelli subcomplanati, opaci, $2 \mathrm{~mm}$. diam.; veteriores cortice griseo opaco vestiti. Folia petiolo canaliculato 10-15 mm. longo; lamina elliptico-oblonga an oblonga, $13-17 \mathrm{~cm}$. longa et $5.7-6.5 \mathrm{~cm}$. lata, apice subacuminata, basi subacuta an subrotundata, subcoriacea, supra nitidula, sicc. supra saturate et subtus dilute olivacea, costa subtus prominente sed haud carinata, nervis utroque latere costae 9-10 subtus prominulis, reticulatione subdensa utrimque distinguenda. Stipulae in vaginam cylindricam connatae, parte libera latissime triangulari, breviter aristata, $2 \mathrm{~mm}$. longa. Inflorescentia foliis normalibus praecessa, subsessilis, laxe trichotome corymboso-paniculata, glabra, floribus circ. 150; pedunculus $3 \mathrm{~mm}$. longus; ramuli trichotomiae primae et secundae basi articulati; internodium basale axis $6-8 \mathrm{~cm}$., internodia basalia ramulorum infimorum 5-7 cm., internodia alia breviora; pedicelli 3-5 mm.; ramuli infimi foliis lineari-lanceolatis sessilibus $1 \mathrm{~cm}$. longis, a stipulis normalibus connatis suffulti; ramuli jugi secundi bracteis minimis, alii ebracteati; bracteolae nullae. Flores glabri. Calyx $0.8 \mathrm{~mm}$. altus, ovario paulo brevior, tubo quam lobis late ovato-triangularibus paulo longiore. Corolla alba, extus intusque glabra, tubo $5 \mathrm{~mm}$. longo, lobis subobtusis $6.5 \mathrm{~mm}$. longis et $2.5 \mathrm{~mm}$. latis. Stamina filamentis $2 \mathrm{~mm}$. longis, antheris apiculatis $5 \mathrm{~mm}$. longis. Stylus parte inclusa dense pilosus, stigmatibus $3 \mathrm{~mm}$. longis comprehensis $10 \mathrm{~mm}$. longus. Drupa globosa, nigra, leviter bisulcata.

Solomon Islands: U l a w a I s l a n d, Ulawa, in rain forest, leg. L. J. Brass 2948, Oct. 1932 (Arnold Arb., Type). S a n C r i s t o v a 1 I s 1 a n d, Star Harbour, Harbour foreshore, leg. L. J. Brass 3094, Oct. 1932 (Arn. Arb.).

This plant resembles $I$. timorensis Decn., but the entirely glabrous corolla throat proves that it belongs to the section Pavetropsis. Its nearest allies are doubtless found among the Fijian species: I. elegans Gillespie and I. tubiflora A. C. Smith especially come very near to it. The style of the latter has been described as glabrous, but this point deserves further study: a few hairs on the included part may have been overlooked. From I. elegans it differs in its glabrous calyx and in the shorter lobes of the latter; from I. tubiflora in the much shorter corolla and in the absence of bracteoles.

Botanical Museum, Utrecht. 


\section{$2 \mathrm{BHL}$ Biodiversity Heritage Library}

Bremekamp, Cornelis Eliza Bertus. 1939. "New Ixora Species from the Solomon Islands." Journal of the Arnold Arboretum 20(2), 216-219. https://doi.org/10.5962/p.185408.

View This Item Online: $\underline{\text { https://www.biodiversitylibrary.org/item/33596 }}$

DOI: https://doi.org/10.5962/p.185408

Permalink: https://www.biodiversitylibrary.org/partpdf/185408

\section{Holding Institution}

Missouri Botanical Garden, Peter H. Raven Library

\section{Sponsored by}

Missouri Botanical Garden

\section{Copyright \& Reuse}

Copyright Status: In copyright. Digitized with the permission of the rights holder.

Rights Holder: Arnold Arboretum of Harvard University

License: http://creativecommons.org/licenses/by-nc-sa/3.0/

Rights: https://biodiversitylibrary.org/permissions

This document was created from content at the Biodiversity Heritage Library, the world's largest open access digital library for biodiversity literature and archives. Visit BHL at https://www.biodiversitylibrary.org. 